\title{
Correction to: The ELFIN Mission
}

V. Angelopoulos ${ }^{1,2} \cdot$ E. Tsai ${ }^{1,2}$ L. Bingley ${ }^{1,2}$ - C. Shaffer ${ }^{1,3}$ - D.L. Turner ${ }^{1,4}$. A. Runov ${ }^{1,2} \cdot$ W. Li ${ }^{1,2,5} \cdot$ J. Liu ${ }^{1,2}$ - A.V. Artemyev ${ }^{1,2} \cdot$ X.-J. Zhang ${ }^{1,2}$. R.J. Strangeway ${ }^{1,2}$ - R.E. Wirz ${ }^{6}$ - Y.Y. Shprits ${ }^{1,7}$ • V.A. Sergeev ${ }^{8}$ - R.P. Caron ${ }^{1,2}$. M. Chung ${ }^{1,4}$ - P. Cruce ${ }^{1,9}$ - W. Greer ${ }^{1,2}$ - E. Grimes ${ }^{1,2}$ - K. Hector ${ }^{1,2,10}$ - M.J. Lawson ${ }^{1,2}$. D. Leneman ${ }^{1,2}$ - E.V. Masongsong ${ }^{1,2}$ - C.L. Russell ${ }^{1,2}$ - C. Wilkins ${ }^{1,2}$ - D. Hinkley ${ }^{11}$. J.B. Blake ${ }^{11}$ - N. Adair ${ }^{1,2,12}$ • M. Allen ${ }^{1,2,9}$ - M. Anderson ${ }^{1,2,13}$ - M. Arreola-Zamora ${ }^{1,2}$. J. Artinger ${ }^{1,2,14}$ - J. Asher ${ }^{1,2,4}$ - D. Branchevsky ${ }^{1,2,11}$ - M.R. Capitelli ${ }^{1,2,12}$. R. Castro ${ }^{1,2,10}$ - G. Chao ${ }^{1,2,15}$ - N. Chung ${ }^{1,2,16}$ - M. Cliffe ${ }^{1,2,17}$ - K. Colton ${ }^{1,2,18}$. C. Costello ${ }^{1,2,19}$ - D. Depe $\mathrm{D}^{1,2,20}$ - B.W. Domae ${ }^{1,2,20}$. S. Eldin ${ }^{1,2,20}$ - L. Fitzgibbon ${ }^{1,2,3}$. A. Flemming ${ }^{1,2,9}$ - I. Fox ${ }^{1,2,6}$ - D.M. Frederick ${ }^{1,2,12}$ - A. Gilbert ${ }^{1,2,20}$ - A. Gildemeister ${ }^{1,2,9}$. A. Gonzalez ${ }^{1,2,17}$ • B. Hesford ${ }^{1,2,21}$ • S. Jha ${ }^{1,2,19}$ • N. Kang ${ }^{1,2,12}$ • J. King,2,19 .

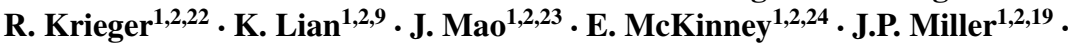
A. Norris ${ }^{1}$ - M. Nuesca1,2,19 - A. Palla1,2,19 - E.S.Y. Park ${ }^{1,2,25}$ - C.E. Pedersen ${ }^{1,2,6}$. Z. Qu ${ }^{1,2,6}$ - R. Rozario ${ }^{1,2,17}$ - E. Rye ${ }^{1,2,20}$ - R. Seaton ${ }^{1,2,6}$. A. Subramanian ${ }^{1,2,9}$. S.R. Sundin ${ }^{1,2,3}$ - A. Tan ${ }^{1,2,26}$ - W. Turner ${ }^{1,2,14}$ - A.J. Villegas ${ }^{1,2,14}$ - M. Wasden ${ }^{1,2,6}$.

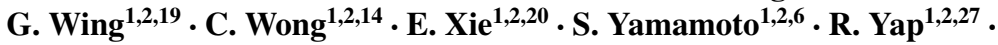
A. Zarifian ${ }^{1,2,21} \cdot$ G.Y. Zhang ${ }^{1,2,28}$

The original article can be found online at https://doi.org/10.1007/s11214-020-00721-7

$凶$ V. Angelopoulos

1 Earth, Planetary, and Space Sciences Department, University of California, Los Angeles, CA 90095, USA

2 Institute of Geophysics and Planetary Physics, University of California, Los Angeles, CA, USA

3 Present address: Tyvak Nano-Satellite Systems, Inc., Irvine, CA 92618, USA

4 Present address: Johns Hopkins University Applied Physics Laboratory, Laurel, MD 20723, USA

5 Present address: Department of Astronomy and Center for Space Physics, Boston University, Boston, MA 02215, USA

6 Mechanical and Aerospace Engineering Department, Henry Samueli School of Engineering, University of California, Los Angeles, CA 90095, USA

7 GFZ German Research Centre for Geosciences, Potsdam, 14473, Germany

8 Saint Petersburg State University, St. Petersburg, 199034, Russia

9 Present address: Northrop Grumman Aerospace Systems, Redondo Beach, CA 90278, USA

10 Present address: Raytheon Space and Airborne Systems, El Segundo, CA 90245, USA

11 The Aerospace Corporation, El Segundo, CA 90245, USA 


\section{Correction to: Space Sci. Rev. (2020) 216: 103 \\ https://doi.org/10.1007/s11214-020-00721-7}

Affiliation 2 in this article is the wrong affiliation that instead should read: "Institute of Geophysics and Planetary Physics, University of California, Los Angeles, CA, USA" which should be regarded as the final version by the reader.

Publisher's Note Springer Nature remains neutral with regard to jurisdictional claims in published maps and institutional affiliations.

12 Present address: Millenium Space Systems, El Segundo, CA 90245, USA

13 Present address: Aptiv, Agoura Hills, CA 91301, USA

14 Physics and Astronomy Department, University of California, Los Angeles, CA 90095, USA

15 Present address: The Boeing Company, Long Beach, CA 90808, USA

16 Present address: SF Motors, Santa Clara, CA 95054, USA

17 Present address: SpaceX, Hawthorne, CA 90250, USA

18 Present address: Planet Labs, Inc., San Francisco, CA 94107, USA

19 Computer Science Department, Henry Samueli School of Engineering, University of California, Los Angeles, CA 90095, USA

20 Electrical and Computer Engineering Department, Henry Samueli School of Engineering, University of California, Los Angeles, CA 90095, USA

21 Present address: Jet Propulsion Laboratory, Pasadena, CA 91109, USA

22 Present address: Mercedes-Benz Research and Development North America, Long Beach, CA 90810, USA

23 Present address: Epic Systems Corporation, Verona, WI 53593, USA

24 Present address: California State Polytechnic University, Pomona, CA 91768, USA

25 Present address: Economics Department, University of California, Los Angeles, CA 90095, USA

26 Present address: Experior Laboratories, Oxnard, CA 93033, USA

27 Mathematics Department, University of California, Los Angeles, CA 90095, USA

28 Present address: Qualcomm, San Diego, CA 92121, USA 